\title{
Prevalencia de factores asociados y complicaciones crónicas en pacientes adultos mayores con Diabetes Mellitus tipo 2 en el Centro de Atención Primaria nivel III EsSalud - El Agustino
}

\author{
Prevalence of associated factors and chronic complications in elderly patients with \\ type 2 diabetes mellitus in the primary care center level III EsSalud - El Agustino
}

\author{
Marielena Aguilar Sánchez¹, Yemim Deisy Maldonado Gonzales², Charo Nátali Huzco Rutti ${ }^{3}$
}

\section{RESUMEN}

Objetivo: Determinar la prevalencia de los factores asociados y las complicaciones crónicas en pacientes adultos mayores con diabetes mellitus tipo 2 en el Centro de Atención Primaria nivel III Es Salud, El Agustino. Materiales y métodos: Estudio descriptivo de tipo cuantitativo y corte transversal. La muestra conformada por 142 pacientes diabéticos adultos mayores seleccionados por muestreo no probabilístico, según criterios de inclusión y exclusión, a quienes se les aplicó un instrumento construido por las investigadoras, probándose su validez y fiabilidad en una prueba piloto a 50 pacientes de características similares. Resultados: El 60.6\% presentó DM 2 como antecedente familiar. Según el índice de masa corporal, el $34.5 \%$ presentó estado nutricional normal, mientras que el $32.4 \%$ presentó sobrepeso y el $29.6 \%$ obesidad; según la circunferencia de cintura, el $68.3 \%$ presentó muy alto riesgo de sufrir enfermedades cardiovasculares y metabólicas. Respecto a la práctica de hábitos saludables, se observó que el $99.3 \%$ no presentó práctica de ejercicio físico y deporte; el $94.4 \%$ presentó inadecuado manejo de la recreación y del tiempo libre; el $99.3 \%$ no consumía alcohol, café ni uso de tabaco; el $67.6 \%$ presentó inadecuados hábitos alimenticios. Finalmente; el $43.7 \%$ presentó infección urinaria y el $35.2 \%$ presentó retinopatía. Conclusiones: El $61 \%$ presentó antecedentes familiares de DM 2, el $62 \%$ presentó sobrepeso y obesidad, el $68 \%$ presentó muy alto riesgo de padecer enfermedades cardiovasculares y metabólicas, el 36\% presentó inadecuado control de la HbA1c ( $\leq 7 \%$ ), el $99 \%$ no realiza ejercicio físico ni deporte, el $68 \%$ presentó inadecuados hábitos alimenticios, las complicaciones crónicas de mayor prevalencia son las infecciones urinarias $(44 \%)$ y retinopatía (35\%); además, los factores asociados a las complicaciones de la DM 2, en su mayoría, son modificables, por tanto debe existir un adecuado control sobre estas variables, el tratamiento de los pacientes adultos mayores debe estar enfocado en estos factores.

Palabras clave: Diabetes mellitus tipo 2, complicaciones, prevalencia.

\section{ABSTRACT}

Objective: To determine the prevalence of associated factors and chronic complications in elderly patients with type 2 diabetes mellitus in the Primary Health Care Center Level III Es Salud, El Agustino. Materials and methods: Descriptive study of quantitative type and cross section. The sample consists of 142 elderly diabetic patients selected by non-probabilistic sampling, according to inclusion and exclusion criteria, to whom an instrument for investigations is applied, proving its validity and reliability in a pilot test to 50 patients of similar characteristics. Results: $60.6 \%$ presented DM 2 as a family history. According to the body mass index, $34.5 \%$ presented normal nutritional status, while $32.4 \%$ presented overweight and $29.6 \%$ obesity; According to the waist circumference, $68.3 \%$ presented a high risk of cardiovascular and metabolic diseases. Regarding the practice of healthy habits, $99.3 \%$ does not apply to physical exercise and sports; $94.4 \%$ presented the management of recreation and free time; $99.3 \%$ do not consume alcohol, coffee or tobacco use; $67.6 \%$ presented inadequate eating habits. Finally; $43.7 \%$ presented urinary infection and $35.2 \%$ presented retinopathy. Conclusions:

\footnotetext{
${ }^{1}$ Asistente Laboratorio de Nutrición. Universidad Peruana Unión, Lima Perú.

${ }^{2}$ Nutricionista, Cinica Good Hope, Miraflores, Perú.

${ }^{3}$ Nutricionista, Empresa ADN SAC, Asesoria en Deporte y Nutrición, Lima, Perú.
} 
$61 \%$ of relatives with DM $2,62 \%$ with overweight and obesity, $68 \%$ with high risk of cardiovascular and metabolic diseases, $36 \%$ with inadequate control of $\mathrm{HbA} 1 \mathrm{c}(\leq 7 \%)$, $99 \%$ without realization. physical exercise and sport, $68 \%$ presented inadequate eating habits, the most prevalent chronic complications are urinary tract infections $(44 \%)$ and retinopathy $(35 \%)$; In addition, the factors associated with the complications of DM 2, for the most part, are modifiable, therefore there must be adequate control over these variables, the treatment of adult adults should be focused on these factors.

Keywords: Diabetes mellitus type 2, complications, prevalence.

\section{INTRODUCCIÓN}

La diabetes mellitus tipo 2 (DM2) es una enfermedad crónico degenerativa que se distingue por la presencia de hiperglucemia, cuando el organismo no puede utilizar de manera eficaz la insulina que produce (1).

La prevalencia de DM2 y sus complicaciones aumentan directamente con la edad. En el 2005, el Centro de Control de Enfermedades de los Estados Unidos reportó que la prevalencia de DM2 diagnosticada en personas de 65 a 74 años de edad fue de $18.5 \%$ (2). En el mismo año, la prevalencia de DM2 en adultos mayores de 60 años fue de $11.4 \%$ en el Perú (3), mientras que en el año 2013, la prevalencia de DM2, en adultos mayores de 65 años, fue de $32 \%$ (4).

La Organización Mundial de la Salud (OMS) describe que entre los factores determinantes, asociados a la presencia de complicaciones de la DM2, se encuentran: antecedentes patológicos tanto familiares como personales (obesidad, sobrepeso, hipertensión arterial alta (HTA), accidente cerebro vascular (ACV)), incremento del índice de masa corporal (IMC), perímetro abdominal, falta de control en los niveles de hemoglobina glucosilada $(\mathrm{HbA} 1 \mathrm{c})$ y glucosa en ayunas; malos hábitos alimenticios (como el inadecuado consumo de carbohidratos), sedentarismo, hábitos nocivos como: el consumo de café, alcohol y uso de cigarrillos (1). Asimismo, Ortiz et al, refieren que los altos niveles de estrés se asocian a altos niveles de (HbA1c) (5).

Alrededor de dos tercios de la población diabética tiene o está en riesgo de sufrir complicaciones crónicas (6), estas constituyen la causa del incremento de la morbimortalidad y representan una gran carga económica para los sistemas de salud, la economía mundial y la familiar; por tanto, dichas complicaciones crónicas traen una mala calidad de vida en la población diabética (1).
Algunas de las complicaciones a las que puede llevar la DM2 son el infarto del miocardio, los accidentes cerebrovasculares, la amputación de miembros inferiores, la neuropatía, insuficiencia renal y la retinopatía diabética que es una causa importante de ceguera por el resultado de un daño prolongado a los pequeños vasos de la retina (1).

En el último informe mundial de la DM2, emitido por la OMS, la prevalencia de retinopatía de cualquier tipo en diabéticos es de $35 \%$, mientras que la prevalencia de retinopatía proliferativa es de un $7 \%$. También se informa que, por lo menos, el $80 \%$ de los casos de insuficiencia renal terminal obedece a la DM2, la hipertensión o una combinación de ambos trastornos (1).

En los adultos mayores diabéticos, la frecuencia de enfermedades cardiovasculares (ECV) suele ser de dos a tres veces más alta que en los adultos mayores que no tienen la patología, un estudio de Salud Cardiovascular menciona que los adultos mayores de 65 años tienen un riesgo de muerte por enfermedad cardiovascular dos veces más, es decir, el riesgo de muerte por complicación coronaria es 2.5 veces más alto. Estos resultados confirman que las personas ancianas con DM2 tienen un mayor riesgo de muerte (1).

En España, la neuropatía es la complicación más frecuente de la DM2, afectando al $50 \%$ de los pacientes; asimismo, la retinopatía diabética afecta al $15-50 \%$ de los pacientes diabéticos; por tanto, la Organización Nacional de Ciegos de España (ONCE) menciona que la DM2 es la tercera patología causante de deficiencia visual, es decir, la DM2 complica la pérdida de visión 20 veces mayor a la de la población no diabética. La nefropatía está presente entre el 3 y el $35 \%$ de los pacientes con DM2. El riesgo de padecer insuficiencia renal es 25 veces más entre las personas que padecen DM2; es decir el 30 al $50 \%$ de estas personas entre 10 y 20 años presentan algún grado de afección renal (7). 
En Latinoamérica, según las estadísticas epidemiológicas, la retinopatía diabética es la causa del $7 \%$ de los casos de ceguera y la DM2 es la causa del $12.9 \%$ de los Infartos de miocardio, la nefropatía es causa del $34.7 \%$ de los casos incidentes y $22.8 \%$ de los casos prevalentes de diálisis (8).

En el Perú, la vigilancia epidemiológica de DM2 realizada el 2012 en 12 hospitales piloto con el registro de cerca de 3,000 diabéticos, reportaron el $30 \%$ de las personas presentaban alguna complicación macro o micro vascular siendo las más frecuentes la neuropatía (21\%), el pie diabético $(6 \%)$, la nefropatía (4\%) y la retinopatía $(2 \%)(9)$.

Por lo tanto, es de suma importancia continuar con las investigaciones relacionadas a la población diabética, por los alarmantes índices de complicaciones, las cuales generan más gasto en la economía nacional, afectando la unidad médica y la salud emocional de las familias.

\section{MATERIALES Y MÉTODOS}

El presente proyecto se realizó en el Centro de Atención Primaria (CAP) III EsSalud que se encuentra ubicado en la Av. Riva Agüero 1638, en el distrito del El Agustino, Lima - Perú.

\section{Muestra}

El tipo de muestreo pertenece a un estudio no probabilístico de tipo intencional, porque los participantes fueron seleccionados de acuerdo al criterio de inclusión y exclusión. El número de participantes fue de 142 pacientes diagnosticados con DM2.

\section{Tipo de estudio}

La presente investigación es de enfoque cuantitativo, porque la recolección de datos se argumenta en la medición. De diseño no experimental, porque no se realizó la manipulación de las variables de estudio. Además, de corte transversal, siendo la recolección los datos de la muestra en un solo momento. Finalmente, de tipo descriptivo porque se estudiaron las características y propiedades de una muestra definida, identificando las variables (10)

\section{Instrumentos}

Se consideró como referencia inicial el instrumento llamado "Estilos de vida saludable"
Originalmente creado por Arrivillaga, Salazar y Gómez (2002) presentando un análisis psicométrico según Alfa de Cronbach de 0.85, siendo el mismo posteriormente considerado para su adaptación Palomares (2014) en la tesis de maestría titulada "Estilos de vida saludables y su relación con el estado nutricional en profesionales de la salud, en Lima - Perú" el cual presenta 48 preguntas, y está dividida en 6 componentes tales como: Condición de Actividad Física y Deporte (4 preguntas), Recreación y Manejo del Tiempo Libre (6 preguntas), Consumo de Alcohol, Tabaco y otras Drogas (6 preguntas), Sueño (6 preguntas), Hábitos Alimenticios (18 preguntas), Autocuidado y Cuidado Médico (8 preguntas), dichas preguntas fueron validadas por un juicio de expertos, conformados por tres personas.

El instrumento tiene el objetivo de evaluar la prevalencia de los factores asociados y las complicaciones crónicas de la diabetes mellitus tipo 2; titulado "Cuestionario de factores asociados y complicaciones crónicas de la diabetes mellitus tipo 2", el mismo que estará conformado por seis factores.

El primer factor son los antecedentes patológicos, dividido en antecedentes familiares ya sean de madre, padre u otro familiar con DM2, HTA y ACV; asimismo, antecedentes personales con (HTA, sobrepeso y obesidad). Seguidamente, el segundo factor es la valoración antropométrica (peso, talla, perímetro de cintura e índice de masa corporal). El tercer factor es la valoración bioquímica con (hemoglobina glucosilada); además, el cuarto factor es la práctica de hábitos saludables que incluye: ejercicio físico (4 preguntas), la recreación y manejo del tiempo (6 preguntas), hábitos nocivos (4 preguntas), hábitos alimenticios (18 preguntas) y, por último, el factor de las complicaciones crónicas de la diabetes mellitus tipo 2 (pie diabético, infección urinaria, ceguera, insuficiencia renal, accidente cardiovascular).

Para el inicio del proceso de recolección de datos, se coordinó con la directora del Centro de Atención Primaria (CAP) III Es Salud del distrito de El Agustino, se identificaron 142 pacientes que cumplían con los criterios de inclusión y exclusión, a los cuales se les realizó el consentimiento informado.

La recolección de datos se realizó a través de un cuestionario adaptado a la muestra y los datos 
de interés de esta investigación. Posteriormente, se recolectaron los datos de la historia clínica y tarjetas de control, tales como antecedentes personales y familiares, valores de hemoglobina glucosilada (el tiempo de antigüedad fue menor a un mes) y complicaciones evidenciadas. Luego se realizó una entrevista personal con los pacientes para completar el cuestionario de hábitos, este método facilitó el menor sesgo de memoria en los pacientes. Por último, se realizó la valoración antropométrica, para la medida del peso se usó una balanza de pie, calibrada y certificada, para este procedimiento los pacientes se encontraban en ayunas y se midió el peso actual, para la medida de la talla se utilizó un tallímetro de madera fijo y para la medida de la circunferencia de cintura se usó un centímetro certificado, para este procedimiento los pacientes se encontraban en ayunas.

Todo el proceso de recolección de datos desde la revisión de historias clínicas, la entrevista personal hasta la evaluación antropométrica se realizó en un tiempo aproximado de 20 a 25 minutos por paciente. Este proceso se realizó durante los meses de diciembre 2017 enero del 2018, cual tuvo a cargo de personas entrenadas y calificadas que evitaron el menor sesgo posible en la recolección de la data.

El instrumento fue validado por un juicio de expertos, conformado por 5 jueces especialistas en la materia; posteriormente, se realizó la aplicación en una muestra piloto de 50 pacientes, con el objetivo de determinar la fiabilidad. La validez del instrumento fue de 1.00 , según la prueba la $\mathrm{V}$ de Aiken. Este resultado nos indica una adecuada validez del instrumento; y la fiabilidad, según el Alfa de Cronbach fue de .739. El resultado indica una adecuada confiabilidad.

\section{Análisis de datos}

Para el análisis de datos se utilizó el programa estadístico SPSS, versión 24 . Se realizó un análisis descriptivo de cada una de las variables, y se expresó mediante tablas de frecuencia y porcentaje.

\section{RESULTADOS}

En la Tabla 1 se observa que el $66.9 \%$ de los pacientes son de género femenino, la edad del $52,1 \%$ de la muestra se encuentra entre los 60 y 69 años. El $71.1 \%$ son jubilados, y el $25.4 \%$ no completó la primaria.

Tabla 1.

Distribución de los datos sociodemográficos de los pacientes diabéticos del Centro de Atención Primaria, El Agustino.

\begin{tabular}{lll} 
Variable & N & $\%$ \\
Género & & \\
$\quad$ Masculino & 47 & 33,1 \\
$\quad$ Femenino & 95 & 66,9 \\
Edad & & \\
$60-69$ años & 74 & 52,1 \\
$70-79$ años & 45 & 31,7 \\
$80-87$ años & 23 & 16,2 \\
Ocupación & & \\
Independiente & 16 & 11,3 \\
Dependiente & 25 & 17,6 \\
Jubilado & 101 & 71,1 \\
Nivel de educación & & \\
Primaria incompleta & 36 & 25,4 \\
Primaria completa & 28 & 19,7 \\
Secundaria incompleta & 15 & 10,6 \\
Secundaria completa & 30 & 21,1 \\
Superior incompleta & 3 & 2,1 \\
Superior completa & 30 & 21,1 \\
Total & 142 & 100,0 \\
\hline
\end{tabular}


Prevalencia de factores asociados y complicaciones crónicas en pacientes adultos mayores con Diabetes Mellitus tipo 2 en el Centro de Atención Primaria nivel III EsSalud - El Agustino

\section{Prevalencia de Factores asociados y complicaciones crónicas en DM2.}

Figura 1.

Descripción del factor antecedente familiar de DM2 en los pacientes diabéticos del Centro de Atención Primaria, El Agustino

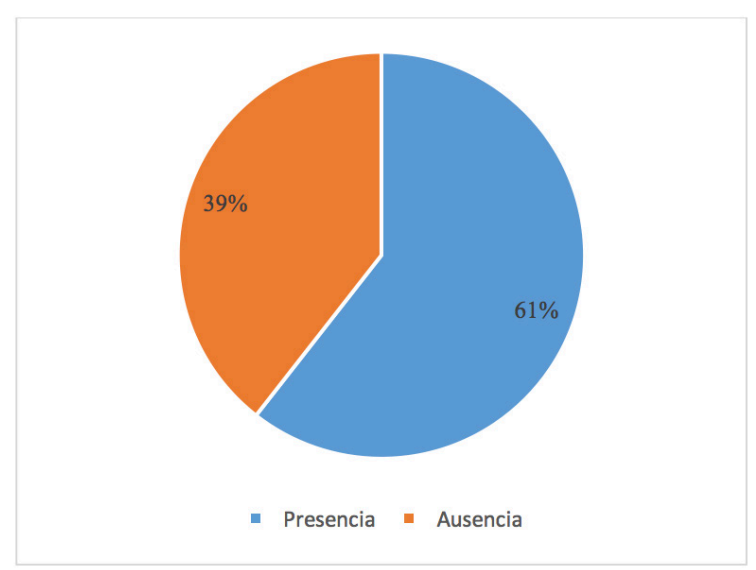

Figura 2.

Descripción del factor antecedente personal de sobrepeso u obesidad en los pacientes diabéticos del Centro de Atención

Primaria, El Agustino.

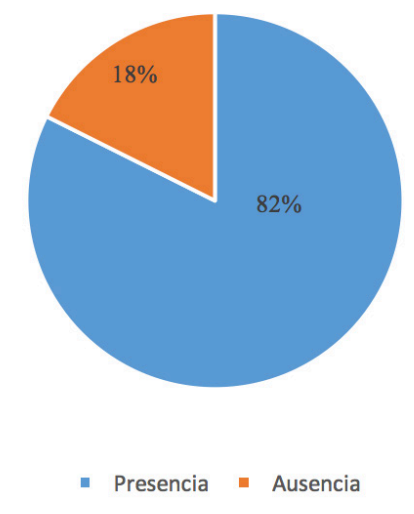

Figura 3.

Descripción del factor antecedente personal de HTA en los pacientes diabéticos del Centro de Atención Primaria, El Agustino

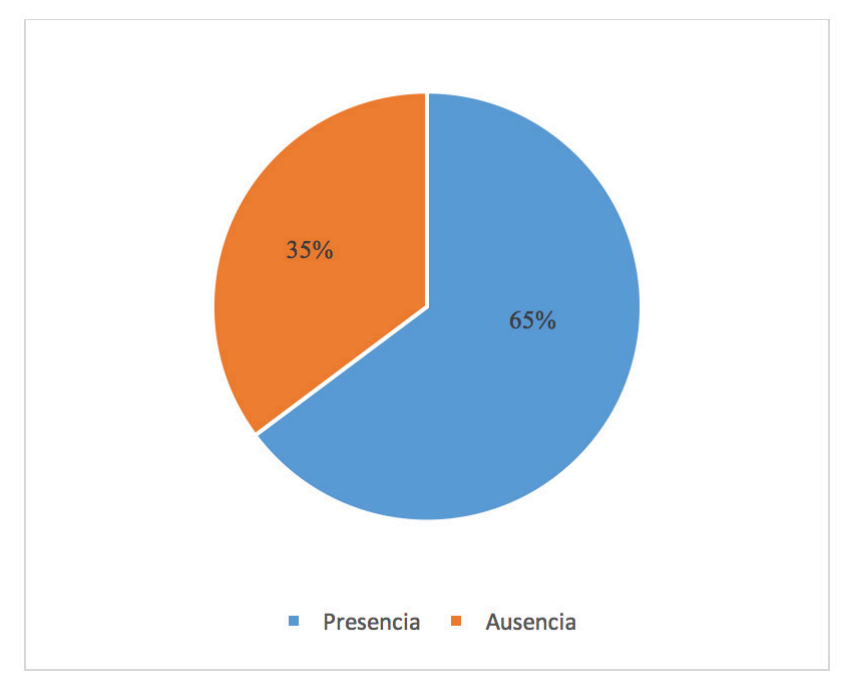


Figura 4.

Descripción de la evaluación del IMC de los pacientes diabéticos del Centro de Atención Primaria, El Agustino

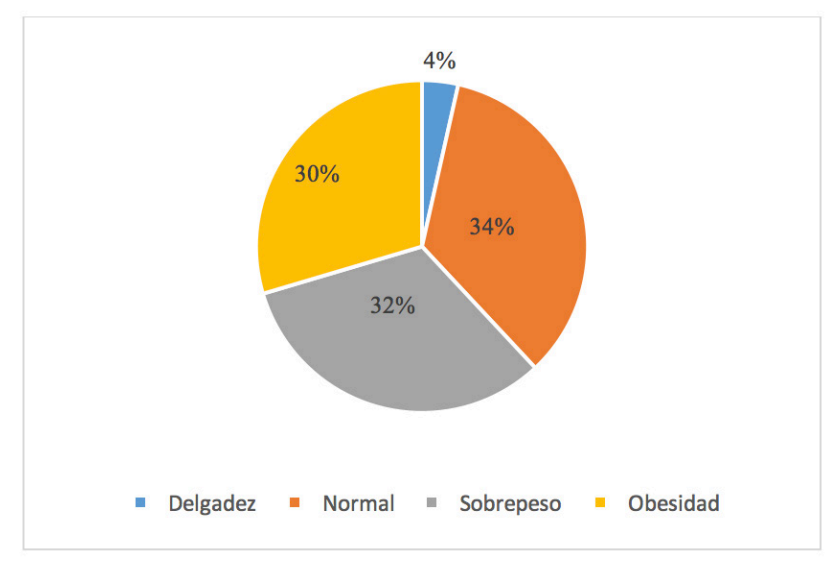

Figura 5.

Descripción de la evaluación del perímetro de cintura en los pacientes diabéticos del Centro de Atención Primaria, El Agustino

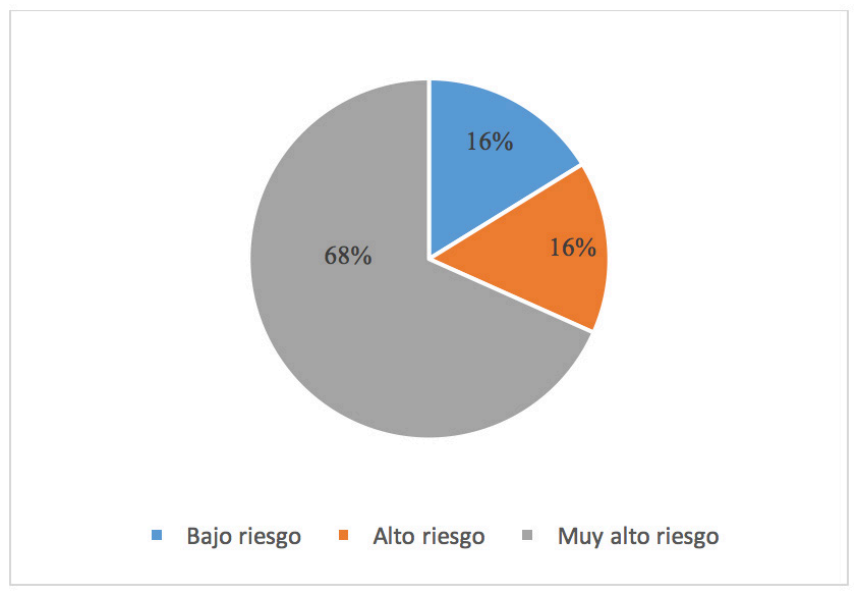

Figura 6.

Descripción de la valoración de la hemoglobina glucosilada en los pacientes diabéticos del Centro de Atención Primaria, El Agustino

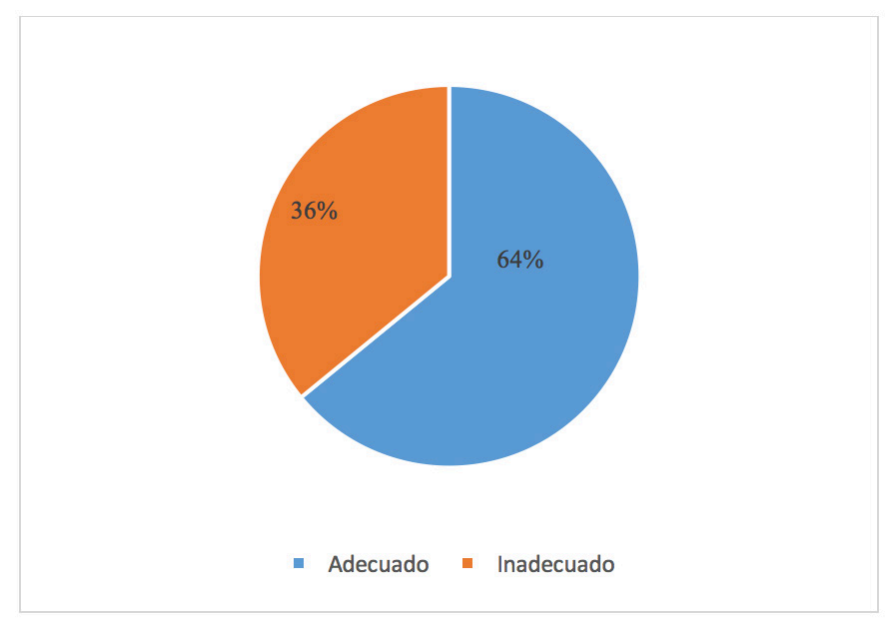


Prevalencia de factores asociados y complicaciones crónicas en pacientes adultos mayores con Diabetes Mellitus tipo 2 en el Centro de Atención Primaria nivel III EsSalud - El Agustino

Figura 7.

Descripción de la práctica de ejercicio físico y deporte en los pacientes diabéticos del Centro de Atención Primaria, El Agustino

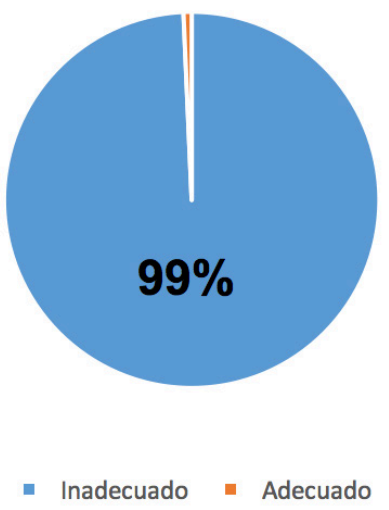

Figura 8.

Descripción de la recreación y manejo del tiempo libre en los pacientes diabéticos del Centro de Atención Primaria, El Agustino

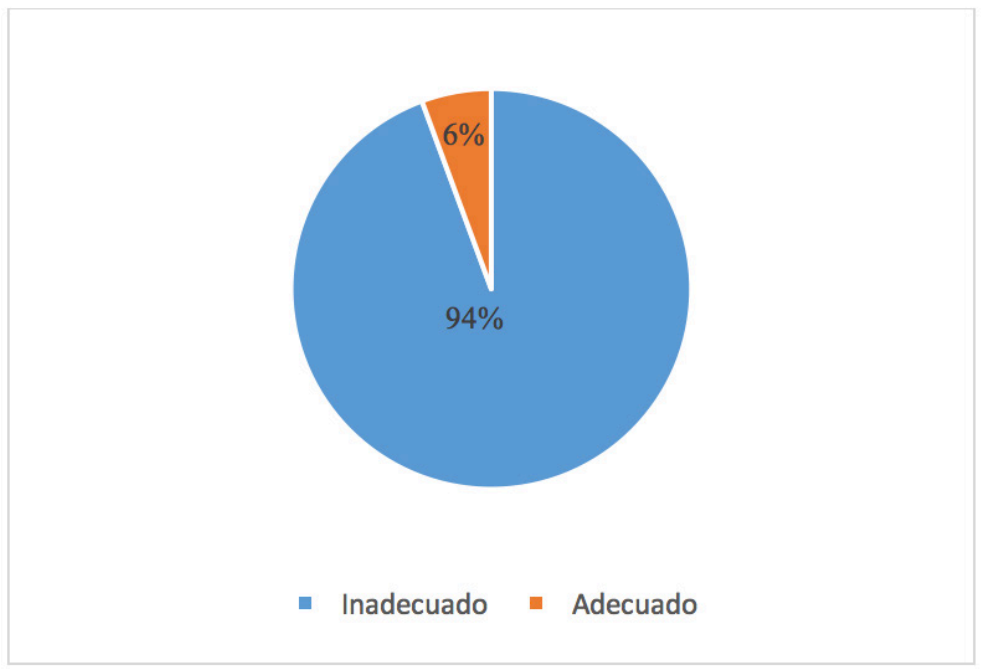

Figura 9.

Descripción de la evaluación del consumo de alcohol, tabaco y café en los pacientes diabéticos del Centro de Atención Primaria, El Agustino

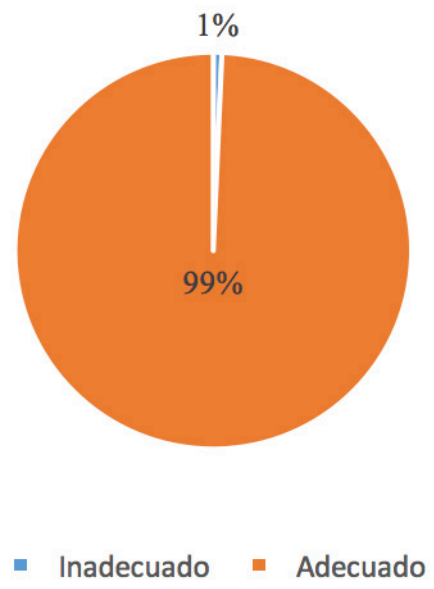


Figura 10.

Descripción de la evaluación de hábitos alimenticios en los pacientes diabéticos del Centro de Atención Primaria, El Agustino

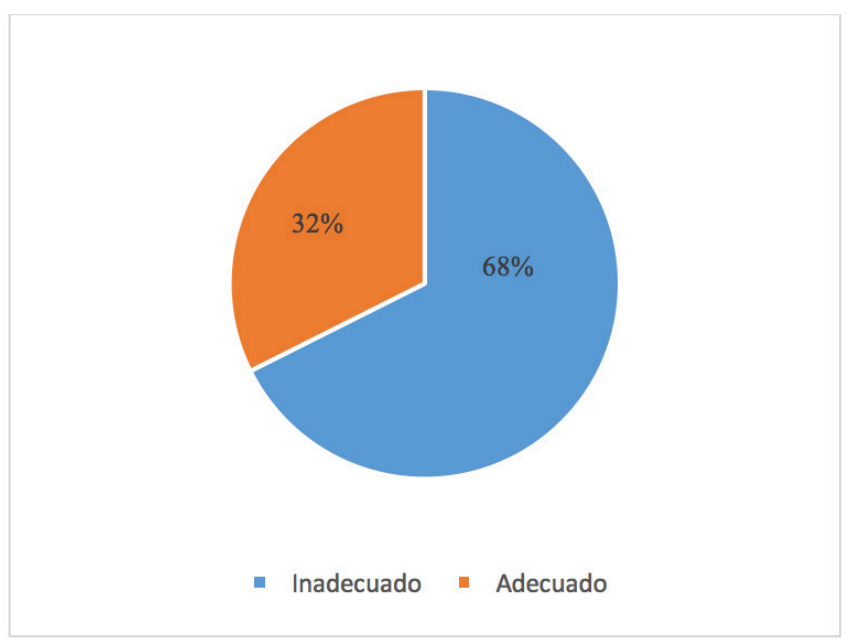

Figura 11.

Prevalencia de complicaciones de la DM2 en los pacientes diabéticos del Centro de Atención Primaria, El Agustino

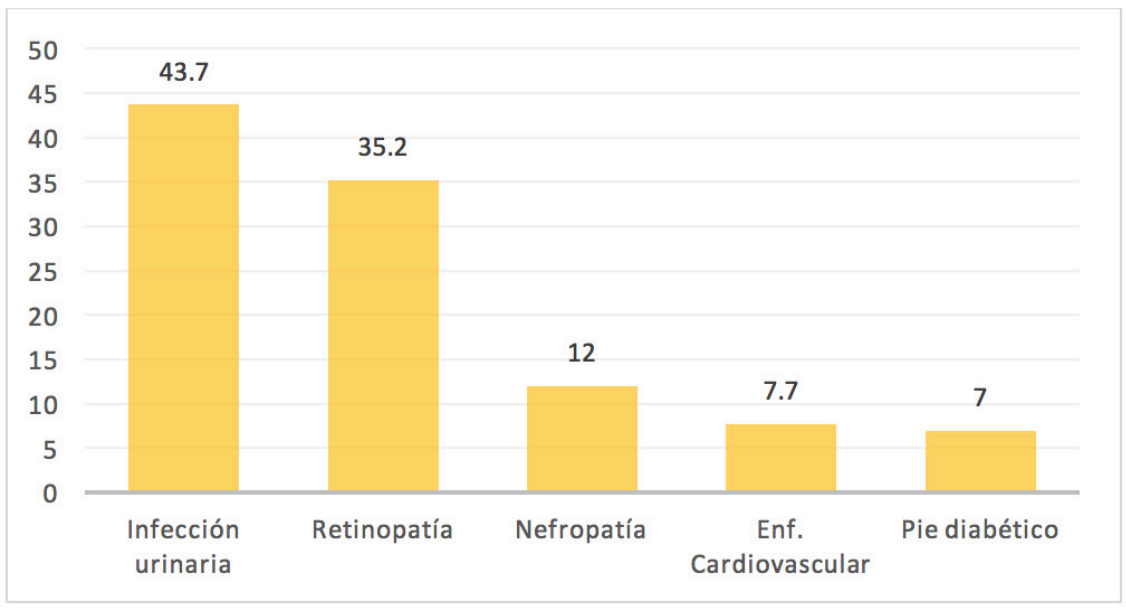

\section{DISCUSIÓN}

Entre los factores de mayor riesgo, sobre las complicacionescrónicas delaDM2, seencuentran los antecedentes patológicos familiares, los resultados de este estudio determinan que el $60.6 \%$ tenía algún familiar diabético (Gráfico 1). Igualmente Noa y Chang (11), en un estudio descriptivo, encontraron que $64.7 \%$ de la muestra presentaba el antecedente familiar de DM2. Por otro lado, Saltos (12) encontró que el $82 \%$ de una muestra de pacientes diabéticos tenían familiares con la enfermedad y que, de estos, el $16.7 \%$ era de relación padre - hijo y el $14.4 \%$ tenía relación madre - hijo, mientras que en este estudio se encontró el $18.3 \%$ y el $17.6 \%$ referente a la relación padre - hijo y madre hijo, respectivamente, sin embargo, la relación más recurrente es la del familiar cercano - paciente $(31.7 \%)$, que incluye hermanos(as) y abuelos (as).

Los antecedentes familiares de DM2 son importantes factores de riesgo sobre la enfermedad, Noa y Chang también mencionan que las personas con historia familiar de DM2 se caracterizan por presentar mayor deterioro de la funcionabilidad de las células beta sobre los diabéticos que no tienen antecedente familiar de DM2, esto tiene como base teórica a la herencia genética. El estudio respalda esta teoría al encontrar que el $60.6 \%$ de la población tiene DM2 como antecedente familiar, asimismo se debe considerar que existe una mezcla entre la carga genética y la práctica de hábitos saludables compartidos. 
Respecto a los antecedentes patológicos personales, este trabajo registra que el $82.4 \%$ presentó sobrepeso u obesidad con anterioridad (Gráfico 2), los datos teóricos informan que existen mecanismos de acción en los que la obesidad actúa como un factor de riesgo para desarrollar resistencia a la insulina y posteriormente DM2; González et al. (13) realizaron un estudio a 40 personas con riesgo de diabetes quienes luego de dos años de seguimiento presentaron DM2, encontrando que el $77,5 \%$ presentaba sobrepeso u obesidad al inicio del seguimiento; esto reafirma la teoría que asocia a la obesidad con la DM2 por medio de la resistencia a la insulina, disfunción de las células beta del páncreas y la falta de control de los niveles de glucosa sérica.

La hipertensión arterial (HTA) ha sido descrita como factor de riesgo para presentar complicaciones de la DM2, en este estudio el $64.8 \%$ de la población presentaba HTA como antecedente personal (Gráfico 3); resultados similares encontraron Valdés y Campos (14) quienes realizaron un estudio descriptivo de corte trasversal, la HTAestuvo presente en el $62 \%$ de la población estudiada, asimismo ellos mencionan que la HTA se asocia a la DM2 debido a la resistencia a la insulina que causa la reabsorción de sodio y agua con aumento de volumen vascular, promoviendo la vasoconstricción y el aumento del angiotensinógeno que activa el sistema renina - angiotensina. De igual manera, Sereday et al. (15) hallaron presencia de HTA en el $63.4 \%$ de la población de un estudio descriptivo. No obstante, Alayón et al. (16) realizaron un estudio descriptivo de corte trasversal y encontraron que la HTA estuvo presente en el $80 \%$ de la población estudiada, resultado que no concuerda con el de este estudio, también de la Paz et al. (17) encontraron presencia de HTA en el $36 \%$ de la población de un estudio descriptivo, este resultado es menor al encontrado en este estudio. Las diferencias entre los resultados presentados pueden deberse a los criterios usados para definir la presencia de HTA, tales como los rangos normales de presión arterial y la presencia o ausencia del uso de medicamentos para el tratamiento de HTA.

En los resultados referente a la valoración antropométrica de la DM2, se observó en la muestra que, según el índice de masa corporal (IMC), el $34.5 \%$ presentó un estado nutricional normal, el $32.4 \%$ presentó sobrepeso, el $29.6 \%$ presentó obesidad (Gráfico 4); según la
Circunferencia de Cintura (CC) el $68.3 \%$ presentó mayor prevalencia de sufrir enfermedades cardiovasculares y metabólicas (Gráfico 5). Asimismo, Quispe (18), en su investigación descriptiva con pacientes diabéticos del hospital III Es Salud Juliaca, encontró que el $40 \%$ de los pacientes presentaron un estado nutricional normal según el IMC, el $26.7 \%$ sobrepeso y el $13.3 \%$ presentó obesidad I y obesidad II; por otro lado, Buendía et al.(19) encontraron que el $76.65 \%$ presentaron circunferencia de cintura elevada; mientras, Zubizarreta et al. (20) evidenciaron que del total de pacientes estudiados, del Hospital Clínico Quirúrgico Hermanos Ameijeirasel, el $67,5 \%$ tenía la circunferencia abdominal aumentada, indicando que existe una mayor prevalencia de obesidad por el aumento de la circunferencia de cintura que por el IMC, lo cual coincide con los resultados de otros estudios que demuestran que la circunferencia de la cintura (CC) identifica más el riesgo cardiovascular que el IMC. Es por eso que, desde el punto vista de evaluación de complicaciones, la medida antropométrica de la CC supera al IMC. No obstante, en una población distinta a la muestra estudiada; Carolino et al. (21), en un estudio descriptivo de 200 participantes adultos de 50 años, encontraron que el $81,33 \%$ los valores del IMC estaba por encima del peso, el $44 \%$ con sobrepeso y $37,33 \%$ con obesidad. Sin embargo, la mayoría de los resultados muestran que los pacientes diabéticos obesos y con circunferencia aumentada tienen mayor prevalencia de padecer complicaciones crónicas.

El control metabólico, a través de la hemoglobina glucosilada, es un factor imprescindible para el seguimiento y prevención de complicaciones de la DM2. En este estudio se observó que el $64.1 \%$ presentó un control metabólico adecuado (HbA1c $\leq 7)$ (Gráfico 6), asimismo, Urbán et al. (22), en un estudio descriptivo, obtuvieron que el $70.4 \%$ tenía niveles controlados de $\mathrm{HbA} 1 \mathrm{c}$, también Zafra et al.(23) describen que el $81.2 \%$ de la muestra, en un estudio descriptivo, presentaban $\mathrm{HbA} 1 \mathrm{c} \leq 7$, considerado como buen control. Por el contrario, Chele (24), en un estudio descriptivo de corte transversal, obtuvo que el $60.5 \%$ de los participantes presentaban valores de hemoglobina glucosilada superior a $7 \%$, lo cual indica un control inadecuado, únicamente el $39.5 \%$ tuvo control adecuado; también Osuna (25), en su estudio descriptivo, obtuvo que el $19 \%$ tenía un buen control metabólico (HbA1c $\leq 7 \%), 49.2 \%$ pobre control metabólico ( $\mathrm{HbA} 1 \mathrm{c}>7 \%$ y $\leq 9 \%)$ y el $31.8 \%$ 
mal control metabólico $(\mathrm{HbA} 1 \mathrm{c}>9 \%)$. Estos dos últimos estudios presentados difieren en sus resultados con los encontrados en el presente estudio. UKPDS (United Kingdom Prospective Diabetes Study) (citado por Osuna) demostró la reducción de presencia de complicaciones de la DM2 (35\%) por cada punto de reducción porcentual, esto reafirma la teoría de la $\mathrm{HbA} 1 \mathrm{c}$ como factor de riesgo para la aparición de complicación y, además, como factor de morbimortalidad en la población diabética. La diferencia de los valores de $\mathrm{HbA} 1 \mathrm{c}$, entre los estudios antes descritos, podría deberse a los distintos hábitos saludables de las poblaciones tales como práctica de hábitos alimenticios, ejercicio físico, entre otros.

En el factor determinante de prácticas de hábitos saludables, asociado a las complicaciones de la DM2, se encuentra el ejercicio físico, los resultados de este estudio descriptivo determinaron que el $99.3 \%$ no presentaba práctica de ejercicio físico y deporte (Gráfico 7); igualmente, De la Paz et al. (17) encontraron, en su estudio descriptivo, que el $56,0 \%$ de la población no practicaban ejercicios; resultados similares encontraron Soares et al. (26) quienes realizaron un estudio descriptivo y evidenciaron que el $65,5 \%$ de la muestra presentó una elevada tasa de sedentarismo, No obstante, Quílez y García (27), en su estudio descriptivo encontraron que el $64,2 \%$ de su muestra practicaba ejercicio físico. Sin embargo, el sedentarismo, es un factor con alta prevalencia en la aparición de ciertas patologías y en especial de la DM2, por lo que se recomienda llevar un estilo de vida saludable con la práctica de ejercicio físico regular. Además, algunos estudios refieren que la práctica de ejercicio físico logra un mejor control glucémico en la DM2, obteniendo niveles de $\mathrm{HbA} 1 \mathrm{c}$ disminuidos. La diferencia de los resultados puede ser por las distintas características, tales como la edad de la población.

El factor relacionado a la recreación y manejo del tiempo libre, asociado a la prevalencia de complicaciones de la DM2, mostraron que el $94.4 \%$ de los resultados de este estudio presentaron una inadecuada práctica en cuanto a la recreación y al manejo de su tiempo libre (Gráfico 8); asimismo, Belendez et al. (28) encontraron en su estudio descriptivo que el $63 \%$ de las personas con DM 2 presentan niveles altos de estrés; también, Ruiz (29) refiere que existe una clara relación entre el estrés y las complicaciones de la DM2; asimismo, describe que el estrés aumenta los niveles de glucosa en sangre. Por el contrario, Fonseca (30) refiere que tener buena comunicación con la familia, recrearse y tener una buena actitud constituyen factores positivos para contribuir con la salud y la disminución de complicaciones crónicas en la DM2.

Entre los factores de hábitos nocivos, determinantes en la prevalencia de complicaciones de la DM2, este estudio descriptivo encontró que el $99.3 \%$ de la población no consumía alcohol, café ni uso de tabaco (Gráfico 9); asimismo, Diez et al. (31) refirieron que el $51,7 \%$ de su población no fumaba ni bebía alcohol. No obstante, De la Paz (17), en un estudio descriptivo, encontró que el $92 \%$ ingería café y tenía una clara relación entre los factores de riesgo en adultos mayores con la DM2. Por el contrario, Salazar et al. (32) describieron que el consumo de café tiene efectos favorables para las complicaciones de la DM2; sin embargo, Carolino et al. (21) en un estudio descriptivo, mencionaron que los hábitos nocivos presentan un alto riesgo de desarrollar complicaciones crónicas en la DM2, también, observaron que el consumo de bebida alcohólica es más frecuente que el uso de tabaco. Las diferencias entre los resultados pueden deberse a diferentes características, tales como la edad y hábitos saludables de la población.

Los hábitos alimenticios han sido descritos como factores de riesgo para presentar complicaciones de la DM2, en este estudio descriptivo muestra que el $67.6 \%$ presentó inadecuados hábitos alimenticios (Gráfico 10); igualmente, De la Paz et al. (17), en un estudio descriptivo con una muestra de 50 adultos mayores con DM2, encontraron que el $56,9 \%$ se alimentaba cada vez que quería, y el resto de la muestra no cubrían sus necesidades de requerimiento; asimismo, Ramos (33) encontró que el $78 \%$ de la muestra tuvo hábitos de alimentación inadecuada; situación similar encontraron Barrera et al. (34) en un estudio descriptivo, transversal, donde observaron que el 42,3 $\%$ prefería alimentos fritos; tenía una ingesta deficiente de proteínas y un $43,4 \%$ consumía una elevada porción de carbohidratos, azúcar y sal. Por tanto, estos resultados reafirman la teoría que los hábitos alimenticios inadecuados favorecen la aparición de complicaciones de la DM2. No obstante, Ramírez et al. (35) realizaron un estudio descriptivo transversal y encontraron que los pacientes diabéticos presentaron un 
$57 \%$ de estilos de vida saludables, debido a que consumen frutas y verduras, consumen una pieza de pan, no comen alimentos entre comidas ni fuera de casa y casi nunca agregan sal ni azúcar a sus alimentos ya preparados. Por lo tanto, se determinó que los pacientes presentan estilos de vida saludables, debido a que la mayoría tiene buenos hábitos nutricionales. La diferencia de los resultados, del factor de hábitos alimenticios, podría deberse a las distintas prácticas de hábitos alimenticios que presenta la población.

Los resultados del estudio, referentes a la presencia de complicaciones de la DM2, mostraron que el $43.7 \%$ presentó la infección urinaria y el $35.2 \%$ presentó retinopatía (Gráfico 11). Asimismo, Pesantez y Ruilova (36), encontraron que la prevalencia para infección urinaria fue de $37.3 \%$; mientras que Zafra et al. (23) encontraron que la prevalencia de retinopatía fue de $30.6 \%$. No obstante, Gutiérrez y Montalvo (37), en un estudio descriptivo de 182 pacientes, encontraron que la complicación más frecuente fue de origen nefrológico $(21,6 \%)$, seguido por la presencia de pie diabético $(18,6 \%)$ y complicaciones oftalmológicas (16,2\%). Respecto a la infección urinaria, González et al. (38) determinaron la prevalencia de $17 \%$ en su estudio descriptivo que incluyó a 300 pacientes diabéticos. Las diferencias entre los resultados puede deberse a las diferentes características de las muestras de los estudios mencionados, tales como la edad, tiempo de evolución de la DM2 e incluso factores genéticos.

\section{CONCLUSIONES}

Los factores de riesgo de padecer complicaciones de la DM2 encontrados en la población estudiada, tales como sobrepeso y obesidad (62\%), circunferencia abdominal aumentada (68\%), control inadecuado de HbA1c (36\%), ausencia de práctica de ejercicio físico y deporte $(99 \%)$ e inadecuados hábitos alimenticios (68\%) son modificables, por tanto, debe existir un adecuado control sobre estas variables y el tratamiento de los pacientes adultos mayores debe estar enfocado en estos factores.

\section{Recomendaciones}

En futuras investigaciones se recomienda correlacionar las variables descritas, para determinar la asociación estadísticamente significativa. Incrementar el tamaño de la muestra, realizar comparativos en muestras de características similares. Incluir variables como tiempo de enfermedad, adherencia y tipo de tratamiento, entre otras. Evaluar la composición corporal de la población mediante medidas tales como pliegues cutáneos, circunferencias, entre otras. Declaración de financiamiento y de conflictos
de interés:

El estudio fue financiado por los autores, quienes declaran no tener conflictos de interés.

\section{Correspondencia}

Marielena Aguilar Sánchez.

Dirección: Universidad Peruana Unión, Carretera central Km 19.5, Lima, Perú

Correo electrónico: marielenaas@upeu.edu.pe

\section{REFERENCIAS}

Organización Mundial de la Salud. Informe mundial sobre la diabetes. 2016.

Mehta R, del Moral M, Aguilar-salinas C. Epidemiología de la diabetes en el anciano. Rev Investig Clínica. 2010;62:305-11.

Villena J. Epidemiología de Diabetes Mellitus en el Perú. Rev Diagnóstico. 2016;55(4):173-83.

Revilla L. Situación de la vigilancia de diabetes en el Perú, al I semestre de 2013. Vol. 22. 2013.

Ortiz M, Gómez D, Ortiz E, Gatica A. Factores psicosociales asociados a la adherencia al tratamiento de la Diabetes Mellitus tipo 2. Rev Ter Psicológica. 2011;29(45):5-11.

Gagliardino JJ, de la Hera M, Siri F. Evaluación de la calidad de la asistencia al paciente diabético en América Latina. Rev Panam Salud Pública. 2001;10(5):309-17.

Goday A. Epidemiología de la diabetes y sus complicaciones no coronarias. Rev Española Cardiol. 2002;55(6):657-70.

Costa JE, Fuente GV. Situación mundial y local de la diabetes. Informe tDNA-PAth. 2012.

Ministerio de Salud del Perú. Guía de práctica clínica para el diagnóstico, tratamiento y control de la Diabetes Mellitus Tipo 2 en el primer nivel de atención. In 2016. p. 64. 
Hernández R, Fernandez C, Baptizta P. Metodología de la investigación. 2006. 839 p.

Noa Ávila LR, Chang Solano M. Factores de riesgo y complicaciones en pacientes con diabetes mellitus tipo 2 en Sucre, 2012. Multimed. 2013;17(2):1-19.

Saltos Solis M. Estilos de vida y factores de riesgo asociados a Diabetes Mellitus Tipo 2. Rev Cienc UNEMI. 2012;8-19.

González R, Perich P, Arranz C. Heterogeneidad de los trastornos metabólicos de las etapas iniciales de la diabetes mellitus 2. Rev Cuba Endocrinol. 2009;20.

Valdés E, Camps M. Características clínicas y frecuencia de complicaciones crónicas en personas con diabetes mellitus tipo 2 de diagnóstico reciente Clinical. Rev Cuba Med Gen Integr. 2013;29(2):121-31.

Sereday M, Damiano M, Lapertosa S. Complicaciones crónicas en personas con diabetes mellitus tipo 2 de reciente diagnóstico. Endocrinol y Nutr. 2008;55(2):64-8.

Alayón AN, Altamar-López D, Banquez-Buelvas C, Barrios- López K. Complicaciones crónicas, hipertensión y obesidad en pacientes diabéticos en Cartagena, Colombia. Rev Salud Pública. 2009;11(6):857-64.

De la Paz K, Proenza L, Gallardo Y, Fernandez $\mathrm{S}$, Mompié A. Factores de riesgo en adultos mayores con diabetes mellitus. Rev Medisan. 2012;16(4):489-97.

Quispe Quispe YR. Evaluación del estado nutricional y sus complicaciones en pacientes diabéticos internados en el hospital III EsSalud Juliaca, Octubre 2014 - Enero 2015. 2015.

Buendía RG, Zambrano M, Morales A, Alejo A, Giraldo L, Gámez D, et al. Perímetro de cintura aumentado y riesgo de diabetes mellitus tipo 2 . Acta Médica Colomb. 2016;41(3):161-2.

Zubizarreta K, Rodríguez N, Castilla AB, Hevia $M$, Becerra N. Relación entre características antropométricas y su repercusión en diabéticos tipo 2 Relation. Rev Ciencias Médicas. 2012;16(2):3-19.

Carolino Regla ID, Molena Fernandes CA, Soares Tasca R, Silva Marcon S, Nakamura Cuman RK. Factores de riesgo en pacientes con diabetes mellitus tipo 2. Rev Latino-am Enferm. 2008;16(2):1-7.
Urbán BR, Coghlan JJ, Castañeda O. Estilo de vida y control glucémico en pacientes con Diabetes Mellitus en el primer nivel de atención. Atención Fam. 2015;22(3):68-71.

Zafra Mezcua JA, Méndez Segovia JC, Novalbos Ruiz JP, Costa Alonso MJ, Faílde Martínez I. Complicaciones crónicas en los pacientes con diabetes mellitus tipo 2 atendidos en un centro de salud. Rev Atención Primaria. 2000;25(8):529-35.

Osuna M, Rivera M, Bocanegra C, Lancheros A, Tovar $\mathrm{H}$, Hernández JI, et al. Caracterización de la diabetes mellitus tipo 2 y el control metabólico en el paciente hospitalizado. Acta Médica Colomb. $2014 ; 39(4)$

Soares AC, Araújo MF, Freitas RW, Zanetti ML, Almeida PC, Damasceno MM. Factores de riesgo para Diabetes Mellitus Tipo 2 en universitarios asociación con variables sociodemográficas. Rev Lat Am Enfermagem. 2014;22(3):484-90.

Quílez P, García MR. Control glucémico a través del ejercicio físico en pacientes con diabetes mellitus tipo 2. Nutr Hosp. 2015;31(4):1465-72.

Beléndez Vázquez $M$, Lorente Armendáriz I, Maderuelo Labrador M. Estrés emocional y calidad de vida en personas con diabetes y sus familiares. Gac Sanit. 2015;29(4):300-3.

Ruiz L. Factores que influyen en el nivel de glucosa. Am Diabetes Assoc. :34-40. Fonseca ME. Actitud positiva contribuye a su salud.

Diez L, Rodríguez F, Banegas JR, Guallar P, Fernández L, Del Rey J. Características demográficas, hábitos de vida e historial del consumo de tabaco de los fumadores ocasionales en España. 2002;281-91.

Salazar BC, Lazcano M. Estrés percibido y adaptación en pacientes con diabetes mellitus tipo 2. 2014;7:77-84.

Ramos W, López T, Revilla L, More L, Huamaní M, Pozo M. Resultados de la vigilancia epidemiológica de diabetes mellitus en hospitales notificantes del Perú, 2012. Rev Peru Med Exp Salud Publica. 2014;31(1):9-15.

Barrera $M$ de $P$, Pinilla $A$, Caicedo LM, Castillo YM, Lozano YM, Rodríguez KM. Factores de Riesgo Alimentarios y Nutricionales en adultos con Diabetes Mellitus. Rev Fac Med Univ Nac Colomb. 2012;60(1):28-40.

Ramírez MM, Carvajalino MA, Coronado MDP, Gómez CA, Mayorga LD, Medina R. Estilo de vida actual de los pacientes con diabetes mellitus tipo 2. Rev Cienc y Cuid. 2011;8(1):21-8. 
Prevalencia de factores asociados y complicaciones crónicas en pacientes adultos mayores con Diabetes Mellitus tipo 2 en el Centro de Atención Primaria nivel III EsSalud - El Agustino

Pesantez C, Ruilova JC. Prevalencia de infección de vías urinarias en pacientes con diagnóstico de Diabetes mellitus tipo 2, ingresados en el departamento de medicina interna del hospital Vicente Corral Moscoso durante el año 2011 y factores asociados. 2013.

Gutiérrez WA, Montalvo CA. Complicaciones crónicas en pacientes con diabetes mellitus tipo 2, en el hospital universitario de Neiva. Rev Fac Salud. 2012;61-72.
Gonzales N, Rodríguez E, Manrique H. Características clínicas y factores asociados a morbilidad intrahospitalaria en los pacientes con diabetes mellitus tipo 2. 2013;26(4):159-65.

Recibido: 06/03/18

Aceptado: 04/05/18 